\title{
La "Píldora en el bolsillo" es una estrategia eficaz en el tratamiento ambulatorio de la fibrilación auricular recurrente de reciente comienzo
}

\section{Objetivo}

Evaluar la factibilidad y seguridad de una monodosis oral ambulatoria de flecainida o propafenona para terminar recurrencias recientes de fibrilación auricular (FA).

\section{Diseño}

Estudio descriptivo de serie de casos, y Antes-Después* para los resultados secundarios. El seguimiento medio fue $15 \pm 5$ meses.

\section{Lugar}

Estudio multicéntrico realizado en Italia.

\section{Pacientes}

Pacientes de 18 a 75 años con recurrencias infrecuentes (1-11 en el año previo) y bien toleradas, con FA de reciente comienzo $(<48$ hs), frecuencia cardíaca $\geq 70 /$ minuto y tensión arterial sistólica $\geq 100$ $\mathrm{mmHg}$ y cardioversión exitosa con monodosis de flecainida $200 \mathrm{mg}$ v.o ó $300 \mathrm{mg}$ (en pacientes $\geq 70 \mathrm{~kg}$ ) o bien propafenona 450 ó 600 mg respectivamente. Criterios de exclusión principales: ECG con pre-excitación o bloqueo de rama o aurículo-ventricular de alto grado, FA previa $\geq 7$ días de duración, cardiopatía isquémica, miocardiopatía dilatada o hipertrófica, Insuficiencia Cardíaca o fracción de eyección $<50 \%$, valvulopatía severa o tratamiento antiarrítmico profiláctico actual.

\section{Intervención}

Para futuras FA, se les informaba que debían tomar la medicación dentro de los 5 minutos de comenzados los síntomas, y concurrir a guardia si las palpitaciones no cedían en 6-8 hs., presentaban disnea, síncope o aceleración de la frecuencia cardíaca.

Medición de los resultados principales

Tasa de conversión a ritmo sinusal y de efectos adversos.
Secundariamente se compararon las internaciones y visitas a guardia en relación al año anterior.

\section{Resultados Principales}

De 268 pacientes consecutivos que recibieron flecainida o propafenona, $58(22 \%)$ se excluyeron por fracaso o efectos adversos. Permanecieron 210 pacientes: flecainida (74) o propafenona (136) para el tratamiento de la "píldora en el bolsillo". El 79\% de los pacientes (165) tuvo un total de 618 episodios de arritmia, de los cuales $569(92 \%)$ fueron tratados 36+/-93 minutos luego del inicio. El tratamiento fue exitoso (palpitaciones interrumpidas dentro de las 6 hs) en 534 episodios (93\%, IC95\% 90 a 95), sin diferencias entre flecainida y propafenona. El tiempo a la resolución de los síntomas de 113+/-84 minutos. De los 165 pacientes con recurrencias, la droga fue efectiva en todos lo episodios en 139 (84\%). Eventos adversos fueron reportados por 12 pacientes (7\%), incluyendo efectos adversos no cardíacos y un paciente con aleteo auricular (AA) de alta respuesta. El número medio de episodios sintomáticos e internaciones mensuales fue significativamente menor luego del estudio que previamente (tabla).

\begin{tabular}{l|c|c|c}
\multicolumn{1}{c|}{ Resultados 2 diarios } & Aĩo previo & Seguimiento & p \\
\hline Episodios sintomáticos & $45,6 / \mathrm{mes}$ & $4,9 / \mathrm{mes}$ & $<0.001$ \\
\hline Internaciones & $15 / \mathrm{mes}$ & $1,6 / \mathrm{mes}$ & $<0.001$ \\
\hline
\end{tabular}

\section{Conclusión}

En una población seleccionada de pacientes con FA recurrente, estratificada por riesgo, la estrategia de "píldora en el bolsillo" es factible y segura, con alta adherencia, baja proporción de eventos adversos y una marcada reducción visitas a guardia e internaciones.

Fuente de financiamiento: Subsidios de la Asociación Italiana de Arritmología y Cardiestimulación.

\section{Comentario}

Muchos pacientes con recurrencias infrecuentes y bien toleradas de FA pero lo suficientemente prolongadas como para requerir terminaciones hospitalarias son los candidatos a beneficiarse con la "píldora en bolsillo".

Los agentes clase IC como la flecainida y la propafenona mostraron en ensayos controlados buena eficacia $(58-95 \%$, y $80 \%$ en este estudio) y rápida acción por v.o en pacientes internados con recurrencias de FA de reciente comienzo, siendo el efecto adverso transitorio más serio el aleteo auricular de rápida respuesta $^{1-7}$. Por ende los autores no se propusieron mostrar la eficacia de la monodosis oral sino su factibilidad y seguridad extrahospitalaria en una población muy bien seleccionada sin cardiopatía relevantes y con una prueba intrahospitalaria previa exitosa y sin complicaciones. Tomando todos estos recaudos la estrategia, que ya era practicada artesanalmente por algunos especialistas, demostró ser factible y en principio segura. Sin embargo se incluyeron sólo $12 \%$ de los candidatos iniciales, limitando la extrapolación de esta estrategia. Si bien la tasa de efectos adversos potencialmente serios, como el AA ocurrió en el $0,6 \%$ debe recordarse que el seguimiento fue de sólo 15 meses y que la repetición de episodios podría incrementar esta cifra aún cuando dentro del hospital no lo presenten. Un meta-análisis ${ }^{8}$ que no incluyó la amiodarona, reveló que la mayoría de las drogas aumenta a mediano plazo la tasa de mortalidad significativamente respecto a los controles, revelando la posibilidad de generar nuevas arritmias (proarritmia). Sin embargo debe destacarse que la mayoría de los pacientes incluidos presentaban cardiopatía orgánica asociada, grupo que debería excluirse categóricamente de cualquier estrategia ambulatoria.

\section{Conclusiones del comentador:}

La cardioversión con una monodosis oral es una importante herramienta para mejorar la calidad de vida de algunos pacientes que padecen esta enfermedad crónica y probablemente también reduzca costos al disminuir considerablemente las visitas a guardia e internaciones, en un pequeño subgrupo de pacientes con FA. Aunque la tasa de complicaciones es muy baja, se requeriría mayor " $n$ " y tiempo se seguimiento para corroborar su seguridad.

\section{Dr. Agustín Ciapponi [ Unidad de Medicina Familiar y Preventiva.Hospital Italiano de Buenos Aires. ]}

* ver glosario

Ciapponi A. La "Píldora en el bolsillo" es una estrategia eficaz en el tratamiento ambulatorio de la fibrilación auricular recurrente de reciente comienzo. Evid. Actual. Pract. Ambul 2005;8:11. Comentado de : Alboni P, Botto GL, Baldi N et al..Outpatient Treatment of Recent-Onset Atrial Fibrillation with the "Pill-inthe-Pocket" Approach. N Engl J Med 2004 351: 2384-2391. PMID 15575054

\section{Referencias}

1. Villani GQ, Rosi A, Piepoli M, et al. The efficacy of oral treatment with flecainide for paroxysmal atrial fibrillation: correlation with plasma concentration. G Ital Cardiol 1990;20:564-568. 2. Boriani G, Capucci A, Lenzi T, Sanguinetti M, Magnani B. Propafenone for conversion of recent-onset atrial fibrillation: a controlled comparison between oral loading dose and intravenous administration. Chest 1995;108:355-358.

3. Azpitarte J, Alvarez M, Baun O, et al. Value of single oral loading dose of propafenone in converting recent-onset atrial fibrillation: results of a randomized, double-blind, controlled study. Eur Heart $\mathrm{J}$ 1997;18:1649-1654.

4. Botto GL, Capucci A, Bonini W, et al. Conversion of recent onset atrial fibrillation to sinus rhythm using a single loading oral dose of propafenone: comparison of two regimens. Int J Cardiol 1997;58:5561.

5. Blanc JJ, Voinov C. Maarek M. Comparison of oral loading dose of propafenone and amiodarone for converting recent-onset atrial fibrillation. Am J Cardiol 1999;84:1029-1032.

6. Khan IA. Single oral loading dose of propafenone for pharmacological cardioversion of recent-onset atrial fibrillation. J Am Coll Cardiol 2001;37:542-547.

7. Capucci A, Villani GQ, Piepoli MF. Reproducible efficacy of loading oral propafenone in restoring sinus rhythm in patients with paroxysmal atrial fibrillation. Am J Cardiol 2003;92:1345-1347.

8. Coplen SE, Antman EM, Berlin JA y col. Efficacy and safety of quinidine therapy for maintenance of sinus rhythm after cardiover-sion. A meta-analysis of randomized control trials. Circulation 1990;82:1106-16. 